\title{
Totally implantable venous access devices in cystic fibrosis: complications and patients' opinions
}

\author{
H.C. Rodgers*, K. Liddle*, S.J. Nixon**, J.A. Innes*, A.P. Greening*
}

Totally implantable venous access devices in cystic fibrosis: complications and patients' opinions. H.C. Rodgers, K. Liddle, S.J. Nixon, J.A. Innes, A.P. Greening. CERS Journals Ltd 1998.

ABSTRACT: The introduction of totally implantable venous access devices (TIVAD) has provided a solution to difficult venous access in patients with cystic fibrosis. Early reports have, however, recognized a number of complications with their use. We report our experience with five devices used over 8 yrs with regard to complications and patient attitudes.

Patients' notes were reviewed to record the details of TIVAD insertion, duration of function, and complications. In January 1996 the surviving 30 patients were surveyed on their attitudes to TIVAD and complications by written questionnaire. Sixty one ports were implanted in 42 patients (aged 16-47 yrs) between June 1988 and January 1996, giving a total of 1,510 patient-months' experience.

The duration of function ranged from 2 weeks to 6 yrs. Survival analysis showed that the median survival of ports was 53 months, 42 out of $61(69 \%)$ had not failed in service at the end of follow-up or patient death. Twenty-three complications occurred in 19 patients. These included: line occlusion (10 patients), venous thrombosis (4), difficult access (3), infection (2), cellulitis (1), inversion of port chamber (2) and pneumothorax (1). The questionnaire showed that patients had strong views on the positioning of their port. Lifestyle issues included interference with seatbelts (8 patients), sport (4), clothing (2), sexual relations (2) and cosmetic appearance (15).

Complication rates were similar to those in other studies, although infection rates and salvage of an occluded port were lower. The survey highlighted a number of lifestyle issues, with cosmetic appearance deemed unsatisfactory by half of the patients. However, the majority ( 28 out of 30 ) believed their totally implantable venous access devices to be a better alternative to cannulae or long lines.

Eur Respir J 1998; 12: 217-220.
*Scottish Adult Cystic Fibrosis Service, and **Surgical Unit, Western General Hospital NHS Trust, Edinburgh, UK.

Correspondence: A.P. Greening

Scottish Adult Cystic Fibrosis Service

Western General Hospital NHS Trust

Edinburgh EH4 2XU

UK

Fax: 441313433989

Keywords: Cystic fibrosis intravenous antibiotic therapy totally implantable venous access devices

Received: June 161997

Accepted after revision February 121998
Recurrent infections in patients with cystic fibrosis, particularly after colonization by Pseudomonas aeruginosa, require repeated courses of intravenous antibiotics. Traditionally, peripheral intravenous cannulae or long lines have been used for this purpose. However, frequent replacements are often required in a single treatment period and the resulting damage to veins makes venous access difficult in the long-term. The introduction of totally implantable venous access devices (TIVAD), also known as "ports", has provided a solution to this problem. They were originally used in cancer patients for the administration of chemotherapy and, compared with long-term percutaneous catheters, were shown to have reduced rates of catheter sepsis [1].

A TIVAD usually consists of a silicone septum mounted in a titanium chamber which is inserted subcutaneously on the anterior chest wall or in the arm. The chamber is connected to a catheter which is tunnelled into a central vein. In Edinburgh, TIVAD are inserted as a day-case procedure, with local anaesthesia, by a surgical team specializing in venous access procedures. Sutures are removed at 7 days. The port chamber is accessed with a Huber needle
(Port-a-Cath $^{\mathrm{TM}}$; Pharmacia, Milton Keynes, UK), the tip of which is designed not to damage the silicone septum. These needles are used to flush the port monthly with Hepflush anticoagulant ( $4 \mathrm{~mL}$ of $\left.100 \mathrm{U} \cdot \mathrm{mL}^{-1}\right)$ and are also available with tubing and connectors for the administration of antibiotics. The Huber needle remains in situ for the duration of the intravenous antibiotic course. Almost all patients, under appropriate instruction from the cystic fibrosis nurse specialist or pharmacist, become self-sufficient in both accessing and flushing their ports and, contrary to earlier reports [2], these systems are widely used for the administration of intravenous antibiotics at home as well as in hospital.

TIVAD have been well accepted by patients, but early reports recognized a number of complications with their use [2-6]. Previous reports have usually described complications in one or two types of TIVAD at each centre. We report here our experience with five devices used in Edinburgh over a period of 8 yrs. The use and complications of TIVAD were systematically examined and patients' attitudes to preoperative and postoperative care and to lifestyle issues were surveyed. 


\section{Materials and methods}

The medical records of all patients who attended the Scottish Adult Cystic Fibrosis Service, Western General Hospital, Edinburgh, and had undergone insertion of a TIVAD were reviewed. The patient and operative data were recorded. The analysis included the patient's age and sex, type of TIVAD, insertion site, surgeon, use of sedation and type of anaesthetic. Data were also collected on the number of TIVAD inserted per patient, the length of function and complications experienced. The surviving patients were surveyed on their attitudes to TIVAD by written questionnaire to determine the reason for port insertion, preoperative concerns including the type of TIVAD, the site of insertion, perioperative concerns including sedation and anaesthesia, problems experienced with ports, routine care, cosmetic appearance and lifestyle issues.

\section{Results}

From June 1988 to January 1996, 61 ports were implanted in 42 patients, aged 16-47 yrs, giving a total of 1,510 patient-months experience. Five varieties of TIVAD were used: Vascuport ${ }^{\mathrm{TM}}$ (Ohmeda, Hatfield, UK), Port-aCath $^{\mathrm{TM}}$ and PAS Port ${ }^{\mathrm{TM}}$ (Pharmacia), Intraport ${ }^{\mathrm{TM}}$ (Fresenius, Hampshire, UK) and Braun (Aylesbury, UK) (table 1). The duration of function ranged from 2 weeks to 6 yrs. Survival analysis indicated that the median survival of ports before irremediable failure was 53 months (fig. 1). In this data set, 30 (of 61) ports were still functioning at the end of the follow-up period, and 12 patients died with functioning ports.

Twenty-three complications occurred in 19 patients, of whom 13 required one replacement port and two required more than two ports. In four patients the ports were salvaged without replacement: following anticoagulant treatment for venous thrombosis (two cases) and after port inversion (two cases). This gives an overall complication rate of 1 in every 65 months of port function. The most frequently observed complications were line occlusion (10 cases out of 61) and venous thrombosis (4 cases). Less frequently experienced complications included infection ( 2 cases), cellulitis ( 1 case), inversion of the port chamber under the skin ( 2 cases), difficult initial access ( 3 cases) and pneumothorax (1 case).

\section{Port occlusion}

Occlusion occurred in 10 cases and presented clinically with pain on the anterior chest wall and difficulty in flus-

Table 1. - Five types of totally implantable venous access device inserted in Edinburgh, 1988-1996

\begin{tabular}{lcrccc}
\hline $\begin{array}{l}\text { Type of } \\
\text { device }\end{array}$ & Dates & $\mathrm{n}$ & $\begin{array}{c}\text { Failed in } \\
\text { service } \\
\mathrm{n}\end{array}$ & $\begin{array}{c}\text { Funct. } \\
\mathrm{n}\end{array}$ & $\begin{array}{c}\text { Funct. } \\
\text { until death } \\
\mathrm{n}\end{array}$ \\
\hline Intraport & $1988-1990$ & 7 & 4 & 1 & 2 \\
Port-a-Cath & $1988-1994$ & 9 & 1 & 4 & 4 \\
Vascuport & $1991-1995$ & 40 & 14 & 20 & 6 \\
PAS Port & $1995-1996$ & 2 & 0 & 2 & 0 \\
Braun & $1995-1996$ & 3 & 0 & 3 & 0 \\
\hline Total & & 61 & 19 & 30 & 12 \\
\hline
\end{tabular}

Funct.: functioning.

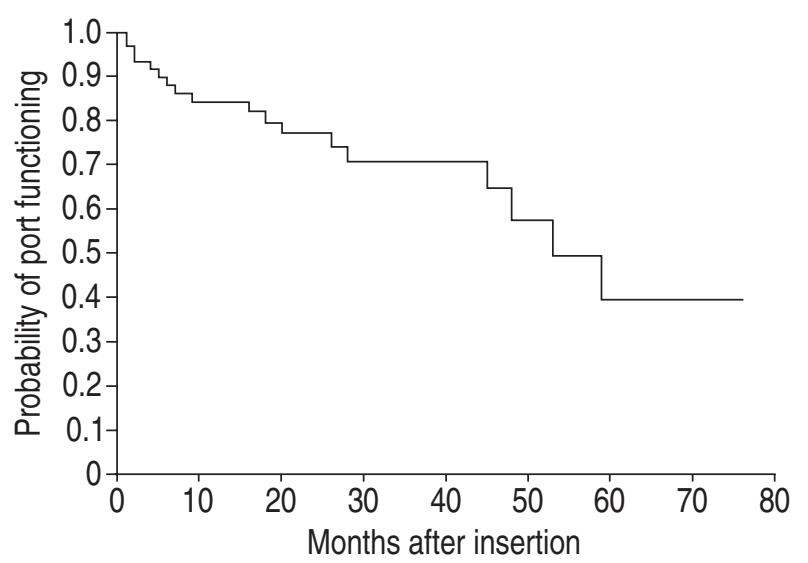

Fig. 1. - Kaplan-Meier survival plot of port function. This plot takes account of "censoring" of the data because of a) patient death with a functioning port or b) variable duration of follow-up in the group whose ports were functioning at the time of writing.

hing the port. The diagnosis was confirmed by injecting contrast into the port ("lin-o-gram"). In three cases no contrast could be injected. In five cases contrast was seen to extravasate from the catheter within the subcutaneous tissue instead of passing through to the tip. It is assumed that the high pressure within the catheter, when flushed with the tip blocked, led to disconnection or rupture of the catheter at its proximal end and subsequent leakage of contrast into subcutaneous tissue. In one case contrast showed the catheter to be kinked at the proximal end. One "lin-o-gram" showed a thrombus at the catheter tip, but venography showed that the vein itself was not occluded by the thrombus.

\section{Port inversion}

In two ports the chamber inverted by $180^{\circ}$ subcutaneously, so that the septum faced inwards, approximately 1 month after port insertion. At that time the local surgical practice was not to suture ports in place subcutaneously, in order to reduce the risk of infection. In both of the above cases the ports were reexposed, repositioned and sutured in place, and one of these was still functioning 20 months later.

\section{Venous thrombosis}

In four patients venography demonstrated a thrombus in a subclavian vein distal to the catheter tip. This presented as pain and swelling of the arm. In each case the port was functioning, as confirmed by "lin-o-gram". All cases were treated with intravenous heparin and three received intravenous streptokinase for 3 days followed by warfarin for at least 3 months. The fourth patient had a severe reaction to streptokinase, with hypotension and rash requiring the drug to be stopped abruptly. In two cases the thrombus did not resolve and the TIVAD was removed and replaced at a different site after several months of anticoagulation. In the other two cases the thrombosis resolved on the above treatment, confirmed by venogram. The TIVAD were still functioning 3 and 49 months later. 


\section{Difficult access}

In two patients access was difficult because of weight gain following nasogastric feeding, and both required replacements. In one of these, a female patient, the TIVAD had been placed within the breast at her request, because of concerns about cosmetic consequences. One patient, a female, had had a port inserted in the groin when attempts at other sites failed, owing to previous port insertions. With positioning in the groin the patient could no longer self-administer antibiotics and access by staff was difficult and embarrassing for the patient. It was finally replaced after 6 months because of these difficulties and a further chest wall port was inserted.

\section{Infection}

There were two cases of infected TIVAD which required replacement. A third patient had a minor episode of local cellulitis which resolved with an oral course of antibiotics.

\section{Miscellaneous}

Two patients had TIVAD removed at the time of lung transplantation, a practice common to most transplant centres because of the risk of infection in patients on long-term immunosuppression. In one patient, however, a further TIVAD was inserted 37 months post-transplant because of recurrent severe episodes of sinusitis requiring intravenous antibiotics. There were also three failed initial insertions. These occurred in cases where the surgeon had less experience in inserting these devices.

\section{Questionnaire}

A $100 \%$ response rate was achieved for the anonym-ous questionnaire to the surviving 30 patients. All TIVAD had been inserted because of difficult intravenous ac- cess. Twenty-two patients (73\%) thought a TIVAD would "make life easier", but three (10\%) viewed it as a backward step, indicating "clinical deterioration". Prior to inser-tion of a TIVAD the patients had been shown the different types available. Twenty-nine (97\%) were happy with the explanation and $26(87 \%)$ remembered seeing a specimen TIVAD. At the time of insertion 11 patients $(37 \%)$ had strong views on the positioning of the device and $10(33 \%)$ on the form of anaesthetic/sedation they were to receive. Seven $(23 \%)$ felt that their views were not taken into account (three were unhappy with positioning, two would have preferred extra sedation and two had a local anaesthetic when their preference had been a general anaesthetic). Twenty-four (82\%) of the patients had had local anaesthesia for the TIVAD insertion.

\section{Lifestyle issues}

Some aspect of cosmetic appearance was unsatisfactory in half of the cases: the scar was too visible (4 cases, $13 \%$ ) or prominent ( 4 cases, $13 \%$ ), the port tubing was visible under the skin (3 cases, 10\%), it interfered with sexual activity ( 2 cases, $7 \%$ ) and the port was visible when wearing certain clothing (2 cases, 7\%). Interference with seatbelts was seen in 8 patients $(27 \%)$, but in 2 cases this was only encountered when the Huber needle was in place for antibiotics. To some this was only a minor inconvenience, but to those who drove for a living it was of greater concern. Interference with sport was reported in 4 cases $(13 \%)$. This was mostly seen in contact sports and 1 patient had to give up Judo. In 2 cases (7\%), both women, the positioning of their port limited the type of clothing worn. The main concern in both cases was that if the port was visible, and therefore noticed by acquaintances or strangers, this would provoke unwanted questions which were embarrassing to answer.

\section{Discussion}

In this study, the complication rate of all TIVAD was $38 \%$, which is comparable to other reports [2-6], with total complication rates of $23-66 \%$, although the infection rate in this study was lower (5\% versus $8-23 \%)$. The most frequent complication was TIVAD occlusion. The most common finding on injection of contrast was extravasation of contrast medium from the catheter in its subcutaneous course. It is presumed this was due to a thrombus at the catheter tip which caused the catheter to split under the high pressure needed to attempt to overcome the block. Although the rate of occlusion, at $16 \%$, was comparable to other studies (9\% [2], 7\% [3], 40\% [4], 11\% [5]) our rate of salvage, once blocked, was low. Other centres have shown that an occluded TIVAD may be saved by injecting urokinase [4] or hydrochloric acid $0.1 \mathrm{M}$ [7] through it, with a potential success rate of up to $96 \%$ [4]. We did not find this possible, owing to rupture or disconnection of the catheter. It is possible that before a device becomes completely occluded it becomes gradually harder and slower to flush, when there may be a period of partial occlusion. Intervention at such a stage may save the device. It would seem sensible to alert patients to seek advice if their TIVAD becomes slow to flush.

Two patients found access difficult following weight gain with nasogastric feeding, which is in contrast to MoRRIS et al. [4] who noted problems with port protuberance in two cases following weight loss. A third patient, whose TIVAD was placed in the groin, found the positioning to be most unsatisfactory both medically and psychologically, and we have subsequently tried to avoid this approach in other patients.

Chamber inversion was seen in two instances, which was felt in part to be due to the practice of not suturing the port in place, in order to reduce the risk of local infection. However, since we have experienced no cases of immediate postoperative infections, we have changed our practice and the chamber is now sutured in place. Port inversion has also been recognized in "twiddlers syndrome" [8], which occurs with manipulation by the patient. This possibility was raised with both patients, who robustly denied that this was the case.

Lifestyle issues relating to these devices have not been widely explored $[9,10]$. The results of the anonymous questionnaire to the surviving 30 patients were reported, although it is possible that the 12 patients who died with 
functioning ports may have had different attitudes towards ports and their complications, which would have biased the results. Problems with cosmetic appearance, which occurred in about half of the cases, contrasted with the study by LILENBERG et al. [10], which found that only $25 \%$ of a group of 80 cancer patients were unhappy with the cosmetic appearance of their TIVAD. However, in that study the patients were significantly older than our cystic fibrosis patients, having a median age of 50 yrs. It may not be surprising that a younger group would be more concerned with cosmetic appearances in general. Of the other issues, the problem with seatbelts has been recognized previously [9], and the optimal site of a chest wall TIVAD depends on whether the patient is mainly the driver or passenger of a car and should be discussed prior to insertion. It is also important to determine whether the lung disease is more severe on one side. If this is the case port insertion is recommended on the opposite side (or consideration of an arm port) so as not to interfere with physiotherapy. These issues aside, we recommend that chest wall port insertion should be over the pectorals on the opposite side to the dominant arm.

In summary, this study has shown that totally implantable venous access devices, although having a significant complication rate, have been well accepted and are the preferred method of venous access in patients with cystic fibrosis.

\section{References}

1. Greene FL, Moore W, Strickland G, Mcfarland J. Comparison of a totally implantable access device for chemo- therapy (Port-A-Cath) and long-term percutaneous catheterization. South Med J 1988; 81: 580-583.

2. Brothers TE, Von Moll LK, Niederhuber JE, Roberts JA, Walker-Andrews S, Ensminger WD. Experience with subcutaneous infusion ports in three hundred patients. Surg Gynecol Obstet 1988; 166: 295-301.

3. Cassey J, Ford WDA, O'Brien L, Martin AJ. Totally implantable system for venous access in children with cystic fibrosis. Clin Pediatr 1988; 27: 91-95.

4. Morris JB, Occhionreo ME, Gauderer MWL, Stern RC, Doershuk CF. Totally implantable vascular access devices in cystic fibrosis: a four year experience with fifty-eight patients. J Pediatr 1990; 117: 82-85.

5. Ball ABS, Duncan FR, Foster FJ, Davidson TI, Watkins RM, Hodson ME. Long-term venous access using a totally implantable drug delivery system in patients with cystic fibrosis and bronchiectasis. Respir Med 1989; 83: 429-431.

6. Yung B, Campbell IA, Elborn JS, Harvey JS, Shale DJ. Totally implantable venous access devices in adult patients with cystic fibrosis. Respir Med 1996; 90: 353-356.

7. Shulman RJ, Reed T, Pitre D, Laine L. Use of hydrochloric acid to clear obstructed central venous catheters. $J$ Parenter Enteral Nutr 1988; 12: 509-510.

8. Gebarski SS, Gebarski AS. Chemotherapy port "twiddlers syndrome". Cancer 1984; 54: 38-39.

9. Davies MJ, Wilson RG, Nixon SJ. Implantable venous access catheters: what the patients say. $J R$ Coll Surg Edinb 1992; 37: 125-126.

10. Lilenberg A, Bengtsson M, Starkhammar H. Implantable devices for venous access: nurses' and patients' evaluation of three different port systems. J Adv Nurs 1994; 19: 2122. 